\title{
Exploring the Impact of Infusion Frequency in Hemophilia A: Exit Interviews with Patients Participating in BAY 94-9027 Extension Studies (PROTECT VIII)
}

\author{
Jane R. Wells ${ }^{1}\left[\right.$. Adam Gater ${ }^{1} \cdot$ Chris Marshall $^{1} \cdot$ Theo Tritton $^{1} \cdot$ Parth Vashi $^{2} \cdot$ Sophia Kessabi $^{3}$
}

Published online: 16 July 2019

(c) The Author(s) 2019

\begin{abstract}
Introduction Prophylactic treatment of severe hemophilia A is burdensome, requiring frequent intravenous injections. Extended half-life (EHL) factor VIII replacement therapies offer longer intervals between infusions while still meeting efficacy and safety outcomes; however, patient perspectives following long-term use of such products in the real-world remain unknown.

Objective We aimed to explore the importance of infusion frequency and the potential benefits of reduced infusion frequency among patients receiving prophylactic treatment with an EHL product (BAY 94-9027).

Methods Patients with severe hemophilia A participating in the PROTECT VIII extension study were invited to participate in a semi-structured, concept elicitation 'exit' interview to discuss their experiences. Participants were recruited from Israel, The Netherlands, and the US. Interview transcripts were translated into English and analyzed using thematic analysis methods. Results Sixteen participants (29-68 years of age) infusing with BAY 94-9027 once every 7 days, once every 5 days, or twice weekly were interviewed. Participants reported infusion frequency (alongside efficacy) as the most important treatment attribute influencing their satisfaction with therapy. Patient-reported benefits of reduced infusion frequency and longer duration of factor coverage included greater ability to participate in physical activities; better vein health; less time to schedule and administer factor VIII; reduced impact on work; and improved emotional well-being.

Conclusions This study provides rich insights into the experiences of patients with EHL products and the value of reduced infusion frequency. Such data could be of value to a range of stakeholders (e.g. regulators, payers) and facilitate patient-clinician discussions to promote tailored treatment decisions.
\end{abstract}

\section{Introduction}

Hemophilia A is a hereditary bleeding disorder characterized by repeated and prolonged bleeds into muscles and joints that result in pain, limitations to physical functioning, and impacts on wider health-related quality of life (HRQoL) $[1,2]$. Caused by missing or defective clotting protein called factor VIII (FVIII), current treatment for hemophilia A focuses on ensuring adequate levels of FVIII in blood plasma introduced via intravenous infusion. Prophylactic treatment

Jane R. Wells

jane.wells@adelphivalues.com

1 Adelphi Values Ltd, Adelphi Mill, Grimshaw Lane, Bollington, Cheshire SK10 5JB, UK

2 Bayer, Whippany, NJ, USA

3 Bayer, Basel, Switzerland

\section{Key Points}

Interviews with men with severe hemophilia A $(n=16)$ provided insights into experiences of a new long-acting treatment (BAY 94-9027) requiring fewer and less frequent intravenous infusions.

Benefits discussed by participants included greater ability to participate in physical activities; better vein health; less time to schedule and administer factor VIII; reduced impact on work; and improved emotional well-being.

This information can be used to support patient-clinician shared decision making and tailored treatment regimens. 
of people with severe hemophilia A with FVIII therapies has been shown to reduce bleeding and improve joint outcomes when compared with on-demand treatment $[3,4]$. However, despite the benefit of prophylaxis, adherence to such regimens is suboptimal in a considerable proportion of patients, increasing the likelihood of life-threatening and debilitating bleeds $[5,6]$. Due to the pharmacokinetic profiles of standard FVIII therapies (notably elimination half-life), prophylactic regimens are burdensome to patients, requiring intravenous injections three to four times per week. Issues related to venous access, time to prepare and administer treatment, and lifestyle factors can contribute to treatment burden and may impact adherence $[1,5]$.

In the continued development of FVIII therapies, extended half-life (EHL) products have emerged, promising longer intervals between prophylactic infusions [7-9] while still meeting efficacy and safety outcomes [10-12]. Such therapies present the possibility of tailoring regimens to individual needs, potentially improving adherence, clinical outcomes and HRQoL among patients [13]. BAY 94-9027 (a site-specific PEGylated recombinant FVIII) is one such therapy that has demonstrated an extended half-life versus standard-acting FVIII products in phase I and phase II/III (PROTECT VIII) studies [10, 14]. Understanding the patient perspective of such products is critical for evaluating the value of EHL products but, at present, direct patient experiences and perspectives following long-term use of such products in the real-world remain unknown.

Regulatory and research communities have endorsed qualitative 'exit' interviews as a means of promoting patient-centric evaluation of treatment benefit [15]. Data from such interviews can be used to supplement data collected via standardized patient-reported outcome (PRO) measures implemented in clinical studies. Exit interviews are considered particularly valuable in the case of novel therapies where treatment effects may not be known or not assessed by existing PRO measures, and rare diseases where small sample sizes may limit the sensitivity of PRO measures and opportunities to capture statistically significant and clinically meaningful changes.

The aim of the present study was to explore the importance of infusion frequency and to obtain direct feedback regarding the potential benefits of reduced infusion frequency among patients receiving treatment with an EHL product (BAY 94-9027).

\section{Materials and Methods}

\subsection{Recruitment}

PROTECT VIII was a phase II/III, multicenter, partially randomized, open-label, 36-week trial designed to assess the safety and efficacy of BAY 94-9027 for on-demand treatment and prophylaxis at various dosing frequencies in patients with severe hemophilia A with no history of inhibitors (ClinicalTrials.gov identifier: NCT01580293). Adult patients aged at least 18 years who were currently participating, or had recently (within the past 3 months) participated, in the optional extension phase of the PROTECT VIII study (i.e. had agreed to continue treatment with BAY 94-9027 for at least 100 total exposure days) were invited to take part in this interview study. Patients receiving regular prophylactic treatment with BAY 94-9027 once every 7 days (E7D), once every 5 days (E5D), or twice per week $(2 \times W)$ were eligible for participation.

Patients were recruited from clinical trial sites in Israel, The Netherlands and the US. Patients in Israel and The Netherlands were approached directly by their clinician, while patients in the US were mailed an invitation letter by post via their clinical site. All patients were provided with written information about the study and written informed consent was obtained.

\subsection{Interview Procedure}

Semi-structured concept elicitation (CE) interviews lasting approximately $30 \mathrm{~min}$ were conducted via telephone by trained qualitative researchers in the local language. The content of the interview guide was informed by existing qualitative literature and was translated from English into the local language (where applicable).

Initial discussions with participants were broad and open-ended to establish rapport and facilitate spontaneous elicitation of concepts relevant to their experience of treatment for hemophilia A ("Talk me through a typical week using your current treatment"). Probing questions were then used to gather information on specific topics of interest once every opportunity for spontaneous elicitation had been provided ("How easy or difficult is it to administer your current treatment?"). Questions were designed to provide insight into participants' evaluation of prior and current treatments for hemophilia (i.e. factors influencing participants' satisfaction with therapy), with targeted questions asked to explore participant perspectives regarding infusion frequency and factor coverage, along with impacts on various aspects of their HRQoL.

Participants were compensated for the time taken to complete the interview. All interviews were audiorecorded, transcribed verbatim and anonymized.

\subsection{Analysis}

Interview transcripts were imported into Atlas.Ti [16], a software package used to facilitate the storage, coding, 
and analysis of qualitative data. The thematic analysis process involved creating and assigning data-driven codes related to the research aim. Each transcript was coded individually. As new codes emerged, transcripts were reread and analyzed to ensure all codes were consistently applied (conducted by both JW and TT). These codes were grouped according to underlying themes [17].

Conceptual/thematic saturation (i.e. the point at which no new codes or themes emerge with the collection of more data) was assessed by dividing transcripts into three approximately equal groups (based on chronological order of interview conduct) and comparing concepts elicited using a stepwise approach $[18,19]$. Saturation was judged to be achieved if no new relevant concepts emerged in the final group of interviews.

\subsection{Ethical Considerations}

This interview study was conducted independent of the PROTECT VIII study procedures. The study was conducted in accordance with the Declaration of Helsinki, and was approved by Copernicus Group, a centralized Institutional Review Board (IRB) in the US (IRB number: ADE1-16-492). Local ethics approval was obtained from each of the respective sites in Israel and The Netherlands.

\section{Results}

\subsection{Sample Characteristics}

Sixteen males with hemophilia A from Israel $(n=9)$, The Netherlands $(n=5)$, and the US $(n=2)$ were interviewed. The majority of participants were on an infusion schedule of once $\operatorname{E5D}(n=11)$, while the remaining were on a schedule of once E7D $(n=3)$ and $2 \times \mathrm{W}(n=2)$ at the time of the interview (or at end of their participation in the PROTECT VIII extension study). Participant demographic and clinical characteristics collected at screening prior to interview are provided in Table 1.

\subsection{Factor VIII Attributes Influencing Treatment Satisfaction}

Throughout the course of the interviews, all participants discussed various FVIII replacement therapy attributes (not specifically in relation to BAY 94-9027) and the influence of these attributes on their satisfaction with therapy. Attributes identified by participants are outlined in Fig. 1 .

Treatment efficacy and frequency of infusion (also encompassing duration of FVIII coverage) were the treatment attributes most commonly (and almost universally) reported by participants $(n=15 / 16)$.

Of those participants reporting infusion frequency, 12 reported infusion frequency to be among the most important aspects of a treatment for hemophilia A:

Q: "What are the most important things to you in a treatment for hemophilia?" "Less impact on my body is nice. So, the frequency helps out, I only have to stick once a week." (02-USA-BAY94-E7D)

"The coagulation stayed in the body for a long time, and that was the most important, that you after a certain time still have enough coagulation in your blood." (04-NL-BAY94-E7D)

Other attributes reported by participants included safety (including risk of adverse effects; $n=6$ ), mode of administration $(n=6)$, convenience of preparation $(n=4)$, and quantity of product needed $(n=2)$.

\subsection{Participant Perspectives on Extended Half-Life and Reduced Infusion Frequency BAY 94-9027}

Reflecting specifically on experiences of BAY 94-9027, the majority of participants $(n=14)$ highlighted the length of time that BAY 94-9027 lasts in the body as being superior compared with other factor replacement therapies. All but one patient $(n=15)$ explained that because of how much longer the factor lasts in their body, they were required to infuse BAY 94-9027 less frequently than other factor replacement therapies:

"I would inject myself, but the [other] factor was not as efficient as the one I take today. I still had bleeds. It doesn't stay in the body properly." (06-ISRL-BAY94E7D)

"It lasts longer in the body. It's safer - better than the old factor and more effective from the perspective of preventing bleeds." (05-ISRL-BAY94-E5D)

Nine participants commented that the factor level is better maintained in the blood over the week when compared with other factor treatments due to the longer half-life of BAY 94-9027:

"I don't have the distraction as much that I used to. Especially, you know, if you have one time a week where you, you feel a little low on your factor level versus two or three times a week, definitely less of a distraction." (02-USA-BAY94-E7D)

"The previous one was a maximum of $48 \mathrm{~h}$. This one is good for 5-7 days. You feel that you are still in relatively good shape even if you don't inject on the fifth day.” (04-ISRL-BAY94-E5D) 
Table 1 Patient demographic and clinical characteristics collected at screening $[n=16]$

\begin{tabular}{|c|c|}
\hline Characteristic & Value \\
\hline Age, years [mean (range)] & $46.56(29-68)$ \\
\hline \multicolumn{2}{|l|}{ Living status, $n(\%)^{\mathrm{a}}$} \\
\hline Live alone & $2(12.5)$ \\
\hline Live with partner only & $9(56.3)$ \\
\hline Live with parents/children/family or friends & $2(12.5)$ \\
\hline Other & $2(12.5)$ \\
\hline Missing data & $1(6.3)$ \\
\hline \multicolumn{2}{|l|}{ Race, $n(\%)$} \\
\hline White & $16(100)$ \\
\hline \multicolumn{2}{|l|}{ Highest level of education, $n(\%)^{\mathrm{a}}$} \\
\hline High school/secondary & $5(31.3)$ \\
\hline Vocational school & $3(18.8)$ \\
\hline Bachelor's degree & $6(37.5)$ \\
\hline Advanced degree & $1(6.3)$ \\
\hline Missing data & $1(6.3)$ \\
\hline \multicolumn{2}{|l|}{ Work status, $n(\%)^{\mathrm{a}}$} \\
\hline Working full- or part-time & $11(68.8)$ \\
\hline Looking for work & $3(18.8)$ \\
\hline Retired & $1(6.3)$ \\
\hline Missing data & $1(6.3)$ \\
\hline Time receiving treatment for Hemophilia A, years [mean (range)] & $40.38(3-68)$ \\
\hline \multicolumn{2}{|l|}{ Received previous factor VIII, $n(\%)$} \\
\hline Yes & $16(100)$ \\
\hline Kogenate FS & $4(25)$ \\
\hline Advate & $1(6.3)$ \\
\hline Refecto & $1(6.3)$ \\
\hline Helixate & $1(6.3)$ \\
\hline Factor VIII recombinant/not specified & $10(62.5)$ \\
\hline \multicolumn{2}{|l|}{ Schedule of previous factor VIII, $n(\%)$} \\
\hline Regular prophylaxis & $16(100)$ \\
\hline Duration of BAY 94-9027 treatment, months [mean (range) ${ }^{\mathrm{a}}$ & $56.7(52-66)$ \\
\hline \multicolumn{2}{|l|}{ Treating with Bay $94-9027$ at time of interview, $n(\%)$} \\
\hline Yes & $13(81.3)$ \\
\hline No & $3(18.8)$ \\
\hline \multicolumn{2}{|c|}{ Times per week prescribed at time of interview/end of extension studies, $n(\%)$} \\
\hline 2 times a week & $2(12.5)$ \\
\hline Every 5 days & $11(68.8)$ \\
\hline Every 7 days & $3(18.8)$ \\
\hline Total bleeds during last 12 months [mean (range)] & $3.81(0-25)$ \\
\hline Joint bleeds during last 12 months [mean (range)] & $3.19(0-23)$ \\
\hline \multicolumn{2}{|l|}{ Patient reported health in general, $n(\%)^{\mathrm{a}}$} \\
\hline Excellent & $1(6.3)$ \\
\hline Good & $4(25)$ \\
\hline Fair & $7(43.8)$ \\
\hline Poor & $3(18.8)$ \\
\hline Missing data & $1(6.3)$ \\
\hline
\end{tabular}

Data are expressed as $n(\%)$ unless otherwise specified

${ }^{a} \mathrm{~A}$ demographic form was not completed for one patient, therefore some demographic characteristics are reflective of 15 patients only (as indicated) 


\begin{tabular}{|c|c|c|c|c|c|c|c|}
\hline & PATIENT ID & EFFICACY & INFUSION FREQUENCY & SAFETY & MODE OF ADMINISTRATION & $\begin{array}{l}\text { CONVENIENCE OF } \\
\text { PREPARATION }\end{array}$ & QUANTITY OF PRODUCT \\
\hline \multirow{2}{*}{$\begin{array}{l}\text { INFUSION TWICE PER } \\
\text { WEEK (2XW) }\end{array}$} & 01-ISRL & & & & & & \\
\hline & 10-ISRL & & & & & & \\
\hline \multirow{12}{*}{$\begin{array}{l}\text { INFUSION ONCE EVERY } \\
\text { FIVE DAYS (ESD) }\end{array}$} & 01-USA & & & & & & \\
\hline & 02-USA & & & & & & \\
\hline & 03-ISRL & & & & & & \\
\hline & 04-ISRL & & & & & & \\
\hline & 05-ISRL & & & & & & \\
\hline & 07-ISRL & & & & & & \\
\hline & 08-ISRL & & & & & & \\
\hline & 09-ISRL & & & & & & \\
\hline & 01-NL & & & & & & \\
\hline & 02-NL & & & & & & \\
\hline & 03-NL & & & & & & \\
\hline & 05-NL & & & & & & \\
\hline \multirow{2}{*}{$\begin{array}{l}\text { INFUSION ONCE EVERY } \\
\text { SEVEN DAYS (E7D) }\end{array}$} & 06-ISRL & & & & & & \\
\hline & 04-NL & & & & & & \\
\hline \multicolumn{2}{|c|}{$\begin{array}{l}\text { TOTAL PATIENTS REPORTING ATTRIBUTE AS } \\
\text { INFLUENCING SATISFACTION WITH THERAPY (N=/16) }\end{array}$} & 15 & 15 & 6 & 6 & 4 & 2 \\
\hline
\end{tabular}

Fig. 1 Treatment attributes reported by participants to be important when evaluating treatment (drivers of treatment satisfaction)

Despite the reduced frequency of infusions, the majority of participants $(n=14)$ reported bleeding less frequently while taking BAY 94-9027 than when taking other therapies. The remaining two participants-both with an infusion schedule of every 7 days-indicated that they experienced no change in the frequency of bleeds experienced.

\subsection{Participant Feedback on the Impact of Infusion Frequency on the Management of Hemophilia}

\subsubsection{Physical Activities}

All participants discussed their ability to participate in daily and physical activities while taking BAY 94-9027, and how this compared with other therapies they have used in the past. Nine participants reported what they considered to be improvements in terms of being more active/less restricted in their general physical activity $(n=5)$, walking/hiking $(n=4)$, swimming $(n=1)$, taking stairs $(n=1)$, playing golf $(n=1)$, and going to the gym $(n=1)$. While all acknowledged that they still have restrictions and limitations due to the long-term consequences of their condition (e.g. joint damage from prior bleeds), participants reported that they were more able to participate in activities because they were less worried or fearful of developing bleeds or severe bleeds due to physical activity $(n=3)$. Two participants reported feeling as though the factor would last in the body, which gave them confidence to engage in physical activities:
"Listen, I walk. I go down stairs. I'm not afraid of having a bleed. The factor lasts in my body." (06-ISRLBAY94-E7D)

"I find that I have more time as I'm not thinking about, boy I've got to slow down now because I might be getting a bleed" (01-USA-BAY94-E5D)

Participants who reported no change in their ability or willingness to participate in physical activities $(n=7)$ reported that this was largely due to existing damage, or restrictions they place on their engagement with certain abilities due to their hemophilia (independent of treatment):

"Not every physical activity is suitable for me in my medical condition. I don't take risks." (05-ISRLBAY94-E5D)

\subsubsection{Vein and Infusion Site Health}

The majority of participants ( $n=12)$ commented on the physical impact of intravenous delivery of FVIII, describing damage to and/or difficulties with veins, including scarring and/or wear to the skin on the arms and to the veins $(n=8)$, difficulties finding a vein $(n=4)$, needing to heal after each injection $(n=3)$, and swelling at the site of each injection $(n=1)$.

Ten participants highlighted the benefit of fewer infusions on vein health, describing that fewer infusions minimized the acute and cumulative long-term damage to the vein and injection site. Three participants added that longer intervals between infusions meant their veins were better able to heal and recover between infusions: 
"In the past they used to hurt more. When I was injecting more frequently. Today I don't feel the pain. They have enough time to heal. I also inject in a different place each time, so there is enough time for them to heal, to recover." (07-ISRL-BAY94-E5D)

\subsubsection{Scheduling and Time to Administer}

Of the eight participants who commented on the scheduling and time taken to administer treatment, the majority $(n=5)$ described how infusing treatment was a normal part of their routine, and/or it is not a problem to schedule or find the time to administer treatment (even if it may be time-consuming):

"You have to free up time for it three times a week. It is time-consuming... Yes, well there was always some time for it. That was no problem." (04-NL-BAY94E7D)

Two participants discussed how finding the time to infuse can be problematic:

"Being out-of-town trying to bring everything with you, and find some time to do it, especially when there were a handful of us guys sharing a - sharing a house, it was annoying." (02-USA-BAY94-E7D)

Despite this, 9 of the 13 participants who compared scheduling and time to administer BAY 94-9027 with existing therapies reported a benefit, describing how it is easier to incorporate infusions into their routine $(n=6)$ and/or that they spent less time in the week preparing and administering infusions $(n=6)$ due to the need for less frequent infusions:

"You know, it is nicer - you don't have to think about it as much. Just once a week. So, I mean it definitely gave me a little bit more time to stay in my routine and get things done...Being able to do more things. I, I don't have to fit that timeframe into infuse again." (02-USA-BAY94-E7D)

Four participants, while acknowledging that they spent less time administering BAY 94-9027, did not perceive this to be a benefit as they did not consider the scheduling and time needed to administer other therapies to be an issue:

"Well it is once now and then it was three times, it saves you half an hour. Well for me it was no problem to administer three times." (03-NL-BAY94-E5D)

\subsubsection{Work}

Minimizing the impact on work was reported as a benefit of BAY 94-9027 by 6 of the 12 participants who discussed the concept, in terms of greater confidence at work $(n=2)$, fewer/no absences from work $(n=2)$, and greater productivity $(n=2)$ :

"I'm going to the gym almost every day. I don't miss work. So yes, I'm dealing much better." (09-ISRLBAY94-E5D)

\subsubsection{Emotional Well-Being}

Four participants discussed the emotional impact in relation to the frequency and duration of coverage of typical treatments. Two of these participants described experiencing anxiety and low confidence due to the duration of treatment effect:

"I had a lot of anxiety...You're taking something that will not last long in your body. $72 \mathrm{~h}$ is not a long time. And even during those $72 \mathrm{~h}$ it's not maintaining the same level, it's declining." (05-ISRL-BAY94-E5D)

A further two participants described that they experience psychological strain/burden that stems from the necessity of having to inject on a frequent basis:

"That had to do with the fact that the multiple times of injecting a week opposed me. That was more of a psychological barrier. It does not really hurt or so, but it's more about that it becomes a must." (05-NLBAY94-E5D)

Most participants $(n=12)$ highlighted a positive change in their emotional and psychological well-being following treatment with BAY 94-9027 compared with other therapies. Participants reported that they felt more confident $(n=7)$, felt less worried $(n=4)$, did not need to think about hemophilia and/or treatment $(n=4)$, had peace of mind/felt more peaceful $(n=4)$, felt less fearful $(n=2)$, felt better psychologically $(n=2)$, felt safer $(n=2)$, felt less annoyed $(n=1)$, and felt calmer $(n=1)$. Participants attributed these positive changes to the factor lasting longer in their body and the coverage provided by the product:

"I am less fearful. Since the drug is in my body for longer, I don't worry so much about when I last injected or how much. So I worry less." (07-ISRLBAY94-E5D)

"I was taking one-third less injections and I felt safer that I was covered. The period of the study was better for me, psychologically speaking. One-third fewer injections is significant." (01-ISRL-OT-2xW)

\subsubsection{Adherence}

Six participants discussed adjusting the prophylactic infusion schedule of other therapies. Reasons cited for 
non-adherence to these schedules were both unintentional [i.e. participants forgetting $(n=2)$ or not being supplied with sufficient quantity of factor $(n=1)$ ], and intentional [i.e. participants seeking to avoid infusions $(n=2)$ and not making the time to infuse during their day $(n=1)]$ :

"You maybe postpone it because you are not looking forward to it. It has a little bit of a mental strain. Ok, yeah. Did you do it on a fixed time or? Well you try to do it on a fixed time, but if you do it Monday evening then you don't have a fixed time, so you keep on putting back the time because you are not looking forward to it." (04-NL-BAY94-E7D)

One patient described how he was not able to remain adherent to a more frequent administration schedule associated with other treatments but is able to adhere to the less frequent administration schedule of BAY 94-9027:

\begin{abstract}
"Multiple times of injecting a week opposed me. That was more of a psychological barrier. It does not really hurt or so, but it's more about that it becomes a must. And if I compare with what it's like now, I inject two times a week now, I can bear it to inject two times a week. Because that is not too often and it is a big advantage for me. Before I used the current treatment, I had bleedings regularly. And now when I use this, like I said, I have those two, three, four times a year so at most. That is a big improvement for me." (05-NLBAY94-E5D)
\end{abstract}

\subsubsection{Conceptual Saturation}

Analysis of conceptual saturation showed that saturation was achieved with no new concepts relevant to the research question emerging spontaneously in the final set of patient interviews.

\section{Discussion}

Shared decision making between patients and clinicians when selecting the most appropriate therapy for treatment of hemophilia has been a focus of recent research literature $[5,20]$. It is therefore increasingly important to understand patient experiences and preferences for treatments, particularly those aspects of treatment that patients consider to be most impactful to their daily lives and HRQoL.

Recent studies utilizing stated-preference and discretechoice research methodologies have highlighted that, alongside efficacy in terms of preventing bleeds, infusion frequency is one of the most important drivers of treatment satisfaction and determinants of treatment preferences among patients with hemophilia A [21-23]. Implementing a novel approach to the exploration of experiences of patients receiving an investigational drug as part of a clinical trial (as opposed to preferences based on hypothetical treatments), findings from the present study serve to further highlight the value of treatments with reduced infusion frequency to patients with hemophilia.

Qualitative feedback among PROTECT VIII participants interviewed as part of this study highlighted that the longer duration of factor coverage and less frequent administration of BAY 94-9027, compared with conventional FVIII replacement therapies, was associated with numerous benefits, including greater ability to participate in physical activities; better vein health; less time to schedule and administer FVIII; reduced impact on work; and improved emotional well-being. The confidence and ability that longer-acting FVIII products afford patients to participate in physical activities are notably important, with recent research indicating that daily activity is important for people with hemophilia of all ages, and almost all people with hemophilia wish they could be more active relative to their current activity levels [24].

When considering the findings from this study, it is necessary to acknowledge a number of limitations. First, as a study conducted independent of the PROTECT VIII study protocol, participation in this qualitative research study (by both study sites and individual participants) was voluntary. With no guarantees regarding patient participation and the investment associated with study set-up and initiation (including ethical approval and translation of study materials), those countries with the greatest proportion of eligible patients were targeted for recruitment (i.e. US, The Netherlands, and Israel). As a consequence, the resulting sample for this study is reasonably small and is limited in terms of ethnic and geographic diversity. Nonetheless, the sample size is consistent with that of other similar qualitative research studies among hemophilia patients [25-27]. Furthermore, conceptual saturation was achieved, indicating that concepts elicited by participants had been fully explored during the interviews and providing assurances that the sample size was sufficient to address the research objectives.

It is also necessary to consider the context in which this research was conducted (i.e. an open-label clinical trial). As an open-label trial, participants were aware of their treatment assignment. While this more closely resembles management of hemophilia in the real-world, participants had still been enrolled in a clinical study for an investigational product and it is possible that aspects of the trial (e.g. selection of participants, trial procedures) could have introduced biases that would not otherwise be present for marketed therapies. Future research looking to characterize the patient experience of marketed EHL products in a real-world setting would therefore further validate and add to current findings. 
Participants in the current study were on a range of administration schedules, including $2 \times \mathrm{W}$, E5D and E7D. Although participants in the PROTECT VIII study were evenly assigned to E5D and E7D treatment arms at randomization $(n=43)$, that a greater proportion of participants in this study were receiving BAY 94-9027 E5D is consistent with the PROTECT VIII study extension phase whereby a proportion of participants in the E7D arm had transitioned to the E5D arm [28]. In addition, dosages and frequency of infusion could be adapted at any time during the extension at the discretion of the investigator. The intent of the current study was not to compare and contrast the experiences of participants on each of these administration schedules, as adequate dosing for any individual patient is based on a combination of pharmacokinetics and bleeding phenotype. Furthermore, $2 \times \mathrm{W}$, E5D, and E7D all represent extended dosing schedules compared with traditional nonEHL products. For this study, in order to compare participants' experiences of BAY 94-9027 compared with traditional nonEHL products, participants were asked to recall their experience prior to enrolment into PROTECT VIII. In most cases, participants had been taking BAY 94-9027 for several years at the time of the interview and, while feedback appeared to be clear and consistent, this time delay may be a source of recall error among participants. Those considering conducting trial exit interviews are recommended to embed these as a trial activity. This would likely enable higher participation rates, alleviating some of the limitations of this study while also providing the opportunity to collect patient experience data at multiple time points throughout the clinical trial (e.g. prior to randomization, following completion of the experimental phase, and during the extension study) and minimizing recall error.

Finally, understanding of the patient perspective is vitally important for facilitating decisions regarding regulatory approval, access, and clinical prescribing. However, EHL regimens may also have implications for other stakeholders, such as caregivers and adolescent/pediatric patients, as well as treating clinicians, whose perspectives and experiences it would be valuable to explore in the future [29].

\section{Conclusions}

In recent years, there has been increasing recognition of the value of patient perspectives for informing treatment decisions. This study provides unique insights into patient experiences regarding tailored FVIII regimens using an EHL product (BAY 94-9027). Findings indicate that infusion frequency and the duration of factor coverage are important drivers of treatment satisfaction and HRQoL among patients with hemophilia A. Patients reported that such therapies enabled and encouraged them to lead more active lives, an important consideration in a condition characterized by physical disability and limitations. Such methods (i.e. 'exit interviews') may be adopted in similar rare conditions to further understand risk-benefit of novel therapies from the patient perspective.

Acknowledgements The authors wish to extend a special thanks to Jennifer Pocoski for her support in initiating and coordinating this study, including her valued input into the design of the study. The study team would also like to thank the people with hemophilia A who participated in the interviews and provided valuable insight into their experience of treatment. Similarly, the team thank the clinical sites that facilitated this interview study.

Author Contributions All authors contributed to the design of the study. JW, AG, CM and TT were responsible for conducting the research, including the development of study documents, data collection, and data analysis and interpretation. SK and PV contributed to the review of the study documents. JW, AG and CM wrote the manuscript and all authors were responsible for reviewing and revising the manuscript, and have given approval for this version to be published.

Data Availability Statement Data generated from this study are not publicly available; additional data may be provided by the authors upon reasonable request.

\section{Compliance with Ethical Standards}

Funding This study and manuscript development was fully funded by Bayer.

Conflict of interest Jane R. Wells, Adam Gater, Chris Marshall, and Theo Tritton are employees of Adelphi Values, the outcomes research consultancy commissioned by Bayer to conduct this study. Parth Vashi and Sophia Kessabi are employees of Bayer. All authors have no further conflicts to disclose.

Open Access This article is distributed under the terms of the Creative Commons Attribution-NonCommercial 4.0 International License (http://creativecommons.org/licenses/by-nc/4.0/), which permits any noncommercial use, distribution, and reproduction in any medium, provided you give appropriate credit to the original author(s) and the source, provide a link to the Creative Commons license, and indicate if changes were made.

\section{References}

1. Flood E, Pocoski J, Michaels LA, Bell JA, Valluri S, Sasanè R. Illustrating the impact of mild/moderate and severe haemophilia on health-related quality of life: hypothesised conceptual models. Eur J Haematol. 2014;93(Suppl 75):9-18.

2. Gringeri A, Ewenstein B, Reininger A. The burden of bleeding in haemophilia: is one bleed too many? Haemophilia. 2014;20(4):459-63.

3. Berntorp E, Shapiro AD. Modern haemophilia care. Lancet. 2012;379(9824):1447-56.

4. National Hemophilia Foundation. MASAC Recommendation concerning prophylaxis (regular administration of clotting factor concentrate to prevent bleeding). MASAC Document \#241. Hemophilia Foundation; 2016. https://www.hemophilia.org/ 
sites/default/files/document/files/241Prophylaxis.pdf: National. Accessed 10 Apr 2019.

5. Thornburg CD, Duncan NA. Treatment adherence in hemophilia. Patient Prefer Adher. 2017;11:1677.

6. Schrijvers LH, Beijlevelt-van der Zande M, Peters M, Lock J, Cnossen MH, Schuurmans MJ, et al. Adherence to prophylaxis and bleeding outcome in haemophilia: a multicentre study. Br J Haematol. 2016;174(3):454-60.

7. Mahdi AJ, Obaji SG, Collins PW. Role of enhanced half-life factor VIII and IX in the treatment of haemophilia. Br J Haematol. 2015;169(6):768-76.

8. Tiede A. Half-life extended factor VIII for the treatment of hemophilia A. J Thromb Haemost. 2015;13(Suppl 1):S176-9.

9. Muczynski V, Christophe OD, Denis CV, Lenting PJ, editors. Emerging therapeutic strategies in the treatment of hemophilia A. Seminars in thrombosis and hemostasis. Stuttgart: Thieme Medical Publishers; 2017.

10. Reding M, Ng H, Poulsen LH, Eyster M, Pabinger I, Shin HJ, et al. Safety and efficacy of BAY 94-9027, a prolonged-half-life factor VIII. J Thromb Haemost. 2017;15(3):411-9.

11. Nogami K, Shima M, Fukutake K, Fujii T, Taki M, Matsushita T, et al. Efficacy and safety of full-length PEGylated recombinant factor VIII with extended half-life in previously treated patients with hemophilia A: comparison of data between the general and Japanese study populations. Int J Hematol. 2017;106(5):704-10.

12. Hartmann J, Croteau SE. 2017 Clinical trials update: innovations in hemophilia therapy. Am J Hematol. 2016;91(12):1252-60.

13. Petrini P, Valentino LA, Gringeri A, Re WM, Ewenstein B. Individualizing prophylaxis in hemophilia: a review. Expert Rev Hematol. 2015;8(2):237-46.

14. Shah A, Coyle T, Lalezari S, Fischer K, Kohlstaedde B, Delesen H, et al. BAY 94-9027, a PEGylated recombinant factor VIII, exhibits a prolonged half-life and higher area under the curve in patients with severe haemophilia A: comprehensive pharmacokinetic assessment from clinical studies. Haemophilia. 2018;24(5):733-40.

15. US Food and Drug Administration. FDA Patient-Focused Drug Development Guidance Series. 2018. https://www.fda.gov/drugs /developmentapprovalprocess/ucm610279.htm. Accessed $10 \mathrm{Apr}$ 2019.

16. Friese S. Qualitative data analysis with ATLAS.ti. Thousand Oaks: Sage; 2014.

17. Braun V, Clarke V. Using thematic analysis in psychology. Qual Res Psychol. 2006;3(2):77-101.
18. Guest G, Bunce A, Johnson L. How many interviews are enough? An experiment with data saturation and variability. Field Methods. 2006;18(1):59-82.

19. Saunders B, Sim J, Kingstone T, Baker S, Waterfield J, Bartlam B, et al. Saturation in qualitative research: exploring its conceptualization and operationalization. Qual Quant. 2018;52(4):1893-907.

20. Athale A, Giguere A, Barbara A, Krassova S, Iorio A. Developing a two-sided intervention to facilitate shared decision-making in haemophilia: decision boxes for clinicians and patient decision aids for patients. Haemophilia. 2014;20(6):800-6.

21. Steen Carlsson K, Andersson E, Berntorp E. Preference-based valuation of treatment attributes in haemophilia A using web survey. Haemophilia. 2017;23(6):894-903.

22. Chaugule SS, Hay JW, Young G. Understanding patient preferences and willingness to pay for hemophilia therapies. Patient Prefer Adher. 2015;9:1623.

23. Furlan R, Krishnan S, Vietri J. Patient and parent preferences for characteristics of prophylactic treatment in hemophilia. Patient Prefer Adher. 2015;9:1687.

24. Skinner MW, O'Hara J, Mathew P, Nugent D. Persons with hemophilia reinforce their desire to be more active: US findings from an international patient survey. In: Poster presented at the 70th National Hemophilia Foundation (NHF) Bleeding Disorders Conference: 11-13 October 2018; Orlando, Florida.

25. Nilson J, Schachter C, Mulder K, Hahn M, Steele M, Hilliard P, et al. A qualitative study identifying the knowledge, attitudes and behaviours of young men with mild haemophilia. Haemophilia. 2012;18(3):e120-5.

26. van Os S, Troop N, Ryder N, Hart D. Adherence to prophylaxis in adolescents and young adults with severe haemophilia $\mathrm{A}$, a qualitative study with patients. Health Psychol Behav Med. 2018;6(1):277-300.

27. Flaherty LM, Schoeppe J, Kruse-Jarres R, Konkle BA. Balance, falls, and exercise: beliefs and experiences in people with hemophilia: a qualitative study. Res Pract Thromb Haemost. 2018;2(1):147-54.

28. Reding MT, Lalezari S, Pabinger I, Enriquez MM, Ducore JM. Decrease in overall and joint bleeding rates with extended-interval dosing: $>4$ years of bay $94-9027$ prophylaxis in the protect VIII extension. Blood. 2018;132:1206.

29. Schwartz CE, Powell VE, Su J, Zhang J, Eldar-Lissai A. The impact of extended half-life versus conventional factor product on hemophilia caregiver burden. Qual Life Res. 2018;27(5):1336-45. 